\title{
Multivariate Empirical Mode Decomposition for Quantifying Multivariate Phase Synchronization
}

\author{
Ali Yener Mutlu and Selin Aviyente \\ Department of Electrical and Computer Engineering, Michigan State University, East Lansing, MI 48824, USA \\ Correspondence should be addressed to Selin Aviyente, aviyente@egr.msu.edu
}

Received 3 August 2010; Accepted 8 November 2010

Academic Editor: Patrick Flandrin

Copyright ( $) 2011$ A. Y. Mutlu and S. Aviyente. This is an open access article distributed under the Creative Commons Attribution License, which permits unrestricted use, distribution, and reproduction in any medium, provided the original work is properly cited.

Quantifying the phase synchrony between signals is important in many different applications, including the study of the chaotic oscillators in physics and the modeling of the joint dynamics between channels of brain activity recorded by electroencephalogram (EEG). Current measures of phase synchrony rely on either the wavelet transform or the Hilbert transform of the signals and suffer from constraints such as the limit on time-frequency resolution in the wavelet analysis and the prefiltering requirement in Hilbert transform. Furthermore, the current phase synchrony measures are limited to quantifying bivariate relationships and do not reveal any information about multivariate synchronization patterns, which are important for understanding the underlying oscillatory networks. In this paper, we address these two issues by employing the recently introduced multivariate empirical mode decomposition (MEMD) for quantifying multivariate phase synchrony. First, an MEMD-based bivariate phase synchrony measure is defined for a more robust description of time-varying phase synchrony across frequencies. Second, the proposed bivariate phase synchronization index is used to quantify multivariate synchronization within a network of oscillators using measures of multiple correlation and complexity. Finally, the proposed measures are applied to both simulated networks of chaotic oscillators and real EEG data.

\section{Introduction}

Studying the dynamics of complex systems is relevant in many scientific fields, from meteorology and geophysics to economics and neuroscience. In many cases, this complex dynamic is to be conceived as arising through the interaction of subsystems which can be observed in the form of multivariate time series reflecting the measurements from the different parts of the system. The degree of interaction of two subsystems can then be quantified using bivariate measures of signal interdependence such as traditional crosscorrelation techniques or nonlinear measures such as mutual information [1]. Recently, tools from nonlinear dynamics, in particular phase synchronization, have received much attention [2,3]. Phase synchronization of chaotic oscillators occurs in many complex systems including the human brain, where synchronization of neural oscillators measured by means of noninvasive measurements such as multichannel electroencephalography (EEG) and magnetoencephalogra- phy (MEG) recordings (e.g., [3, 4]) is of crucial importance for visual pattern recognition and motor control.

Classically, synchronization of two periodic nonindentical oscillators is understood as adjustment of their rhythms, or appearance of phase locking which is defined as $\phi_{n, m}(t)=$ $\left|n \phi_{1}(t)-m \phi_{2}(t)\right| \bmod 2 \pi<$ constant, where $n$ and $m$ are some integers, and $\phi_{n, m}$ is the generalized phase difference and $\bmod 2 \pi$ is used to account for the noise-induced phase jumps. The first step in quantifying phase synchrony between two time series is to determine the phase of the signals at a particular frequency of interest. Two closely related approaches for extracting the time and frequency-dependent phase of a signal have been proposed. In both cases, the original signal $x(t)$ is transformed with the help of an auxiliary function into a complex-valued signal, from which an instantaneous value of the phase is easily obtained. The first method employs the Hilbert transform to get an analytic form of the signal and estimates instantaneous phase directly from its analytic form [3]. The second approach computes a 
time-varying complex energy spectrum using the continuous wavelet transform (CWT) with a complex Morlet wavelet [4]. The Morlet wavelet has a Gaussian modulation both in the time and in the frequency domains, and therefore it has an optimal time and frequency resolution [5]. It has been observed that the two approaches are similar in their results [6]. The main difference between them is that the Hilbert transform is actually a filter with unit gain at every frequency [2], so that the whole range of frequencies is taken into account to define the instantaneous phase. Therefore, if the signal is broadband it is necessary to prefilter it in the frequency band of interest before applying the Hilbert transform in order to get a proper value of the phase (e.g., [7-9]). Thus, the Hilbert transform approach relies on the a priori selection of band-pass filter cutoffs making the analysis sensitive to changes in experimental conditions. On the other hand, the wavelet function is nonzero only for those frequencies close to the frequency of interest (center frequency), $\omega_{0}$, thus making this approach equivalent to band-pass filtering $x(t)$ at this frequency. However, the wavelet transform-based synchrony estimates suffer from time-frequency resolution tradeoff, that is, the frequency resolution is high at low frequencies and low at high frequencies.

In this paper, we propose to use a recently developed transform, multivariate empirical mode decomposition (MEMD), for quantifying the phase synchrony between multiple time series. EMD is a fully adaptive, data-driven approach that decomposes a signal into oscillations inherent to the data, referred to as intrinsic mode functions (IMFs). Finding the IMFs is equivalent to finding the band-limited oscillations underlying the observed signal. After the IMFs are extracted, the Hilbert transform can be used to obtain highly localized phase information. Thus, EMD can act as a prefiltering tool for the Hilbert transform-based phase synchrony analysis. In previous applications of EMD to phase synchrony analysis of multivariate data $[10,11]$, the IMFs for each time series were extracted individually and were compared individually against the IMFs from the other time series for computing phase synchrony. This approach has multiple shortcomings. First, the IMFs from the different time series do not necessarily correspond to the same frequency, thus making it hard to compute exact within-frequency phase synchronization. Second, the different time series may end up having a different number of IMFs which makes it hard for matching the different IMFs for synchrony computation. Finally, it has been shown that univariate EMD is not robust under noise and may suffer from mode mixing [12]. Recently, extensions of EMD to the field of complex numbers have been developed including complex empirical mode decomposition [13], rotation invariant empirical mode decomposition (RIEMD) [14], and bivariate empirical mode decomposition (BEMD) [15]. These complex extensions of EMD decompose data from different sources simultaneously. It has been shown that the IMFs obtained in this fashion are matched, not only in number, but also in frequency, overcoming problems of uniqueness and mode mixing [16]. The idea of using bivariate EMD to compute phase synchrony between two signals was first suggested in [12], and the BEMD was shown to perform better than univariate EMD for quantifying bivariate synchrony. In many real life systems, the system is composed of multiple subsystems, and bivariate EMD would be inadequate for quantifying pairwise synchrony between the subsystems, since the bivariate EMD of different pairs will result in different number of IMFs with different frequencies making it difficult to compute synchrony at the same frequency for all pairs. The recent extension of BEMD to the trivariate [17] and multivariate cases [18], makes it possible to quantify pairwise phase synchrony across multiple signals. In this paper, we will employ the multivariate EMD proposed in [18] for quantifying multivariate phase synchronization.

The current application of bivariate measures to multivariate data sets with $N$ time series results in an $N \times N$ matrix of bivariate indices, which leads to a large amount of mostly redundant information. Therefore, it is necessary to reduce the complexity of the data set in such a way to reveal the relevant underlying structures using multivariate analysis methods. Recently, different multivariate analysis tools have been proposed to define multivariate phase synchronization. The basic approach used for multivariate phase synchronization is to trace the observed pairwise correspondences back to a smaller set of direct interactions using approaches such as partial coherence adapted to phase synchronization [19]. Another complementary way to achieve such a reduction is cluster analysis, a separation of the parts of the system into different groups, such that the signal interdependencies within each group tend to be stronger than in between groups [20, 21]. Allefeld and colleagues have proposed two complementary approaches to identify synchronization clusters and applied their methods to EEG data [22-25]. In [22], a mean-field approach has been presented which assumes the existence of a single synchronization cluster that all oscillators contribute to a different extent. The authors define the to-cluster synchronization strength of individual oscillators to identify multivariate synchronization. This method has the disadvantage of assuming a single cluster and thus cannot identify the underlying clustering structure. In [23], an approach that addresses the limitation of the single cluster approach has been introduced using methods from random matrix theory. This method is based on the eigenvalue decomposition of the pairwise bivariate synchronization matrix and appears to allow identification of multiple clusters. Each eigenvalue greater than 1 is associated with a synchronization cluster and quantifies its strength within the data set. The internal structure of each cluster is described by the corresponding eigenvector. Combining the eigenvalues and the eigenvectors, one can define a participation index for each oscillator and its contribution to different clusters. This method assumes that the synchrony between systems belonging to different clusters, that is, betweencluster synchronization, is equal to zero and requires an adjustment for proper computation of the participation indices in the case that there is between-cluster synchronization. Despite the usefulness of eigenvalue decomposition for the purposes of cluster identification, it has recently been shown that there are important special cases, clusters of similar strength that are slightly synchronized to each other, 
where the assumed one-to-one correspondence of eigenvectors and clusters is completely lost [26]. Other alternative measures that quantify multivariate relationships include the directed transfer function and Granger causality defined for an arbitrary number of channels [27, 28]. Both of these methods have been applied to study interdependencies and causal relationships; however, they are limited to stationary processes and linear dependencies.

In this paper, the goal is to extend measures of correlation for multiple variables from statistics for quantifying multivariate synchronization. The proposed measures will depend on quantities such as multiple correlation and $R^{2}$ and will be redefined in the context of phase synchrony. In particular, $R_{v}$, a measure of association for multivariate data sets introduced in [29] will be used to quantify the degree of association or synchronization between groups of variables. $R_{v}$ is a particularly attractive measure for quantifying the similarity between groups of variables, since it has been shown to be a unifying metric that when maximized, with relevant constraints, yields the solutions to different linear multivariate methods including principal component analysis, canonical correlation analysis, and multivariate linear regression [29]. A second measure, a global complexity measure based on the spectral decomposition of the bivariate synchronization matrix similar to the $S$ measure defined in [30], will be used to complement the findings of $R_{v}$ by quantifying the synchronization within a network.

The contributions of this paper are twofold. First, multivariate EMD will be used for the first time to define pairwise synchrony between multiple time series across the same frequency. This approach will allow us to quantify the synchrony across data-driven modes/frequencies that are consistent across all of the signals. Second, this paper will extend the notion of bivariate synchrony to multivariate synchronization by employing measures of multivariate correlation and complexity to quantify the synchronization within and across groups of signals rather than between pairs. This approach will be useful for applications such as EEG signals, where the synchronization within or across regions is more important than individual pairwise synchrony.

\section{Background}

2.1. Background on Phase and Synchrony. Synchrony measures the relation between the temporal structures of the signals regardless of signal amplitude. It is well known that the phases of two coupled nonlinear oscillators may synchronize even if their amplitudes remain uncorrelated, a state referred to as phase synchrony. The amount of synchrony between two signals is usually quantified by estimating the instantaneous phase of the individual signals around the frequency of interest. As mentioned earlier, the two main current approaches to isolating the instantaneous phase of the signal are Hilbert transform and complex wavelet transform. In the Hilbert transform method, the signal is first bandpass filtered around the frequency of interest, and then the instantaneous phase is estimated from the analytic form of the signal. In the wavelet transform approach, the phase of the signal is extracted from the coefficients of the wavelet transform at the target frequency, which is basically equivalent to estimating the instantaneous spectrum around a frequency of interest. In both methods, the goal is to obtain an expression for the signal in terms of its instantaneous amplitude, $a(t)$ and phase $\phi(t)$ at the frequency of interest as follows:

$$
\tilde{x}(t, \omega)=a(t) \exp (j(\omega t+\phi(t))) .
$$

This formulation can be repeated for different frequencies, and the relationships between the temporal organization of two signals, $x$ and $y$, can be observed by their instantaneous phase difference:

$$
\Phi_{x y}(t)=\left|n \phi_{x}(t)-m \phi_{y}(t)\right|,
$$

where $n$ and $m$ are integers that indicate the ratios of possible frequency locking. Most studies focus on within-frequency synchronization, that is, the case where $n=m=1$.

Once the phase difference between two signals is estimated, it is important to quantify the amount of synchrony. The most common scenario for the assessment of phase synchrony entails the analysis of the synchronization between pairs of signals. In the case of noisy oscillations, the length of stable segments of relative phase gets very short; further, the phase jumps occur in both directions, so the time series of the relative phase $\Phi_{x y}(t)$ looks like a biased random walk (unbiased only at the center of the synchronization region). Therefore, the direct analysis of the unwrapped phase differences $\Phi_{x y}(t)$ has been seldom used. As a result, phase synchrony can only be detected in a statistical sense. Two different indices have been proposed to quantify the synchrony based on the relative phase difference, that is, $\Phi_{x y}(t)$ is wrapped into the interval $[0,2 \pi)$, and can be summarized as follows.

(1) Information theoretic measure of synchrony: This measure studies the distribution of $\Phi_{x y}(t)$ by partitioning the interval $[0,2 \pi)$ into $L$ bins and comparing it with the distribution of the cyclic relative phase obtained from two series of independent phases. This comparison is carried out by estimating the Shannon entropy of both distributions, that is, that of the original phases, and that of the independent phases, $\rho=\left(S_{\max }-S\right) / S_{\max }$, where $S$ is the entropy of the distribution of $\Phi_{x y}$ and $S_{\max }$ is the maximum entropy for the same number of bins, that is, the entropy of the uniform distribution. Normalized in this way, $0 \leq \rho \leq 1$.

(2) Phase Synchronization Index: This index is also known as mean phase coherence, $\gamma=\sqrt{\left\langle\cos \left(\Phi_{x y}(t)\right)\right\rangle^{2}+\left\langle\sin \left(\Phi_{x y}(t)\right)\right\rangle^{2}}=\mid(1 /$ N) $\sum_{k=0}^{N-1} e^{j \Phi_{x y}\left(t_{k}\right)} \mid$, where the brackets denote averaging over time. It is a measure of how the relative phase is distributed over the unit circle. If the two signals are phase synchronized, the relative phase will occupy a small portion of the circle and 
mean phase coherence is high. This measure is equal to 1 for the case of complete phase synchronization and tends to zero for independent oscillators. This measure can be applied either taking time averages of the phase differences or taking averages over multiple realizations of the same process.

In this paper, we will employ the second measure of phase synchrony, since it is more robust against noise and does not require the estimation of entropy as in the first index.

2.2. EMD. Empirical mode decomposition is a data-driven time-frequency technique which adaptively decomposes a signal, by means of a process called the sifting algorithm, into a finite set of AM/FM modulated components, referred to as intrinsic mode functions (IMFs) [31]. IMFs represent the oscillation modes embedded in the data. By definition, an IMF is a function for which the number of extrema and the number of zero crossings differ by at most one, and the mean of the upper and lower envelopes is approximately zero. The EMD algorithm decomposes the signal $x(t)$ as $x(t)=\sum_{i=1}^{M} C_{i}(t)+r(t)$, where $C_{i}(t), i=1, \ldots, M$ are the IMFs, and $r(t)$ is the residue. The IMF algorithm can be described as follows.

(1) Let $\tilde{x}(t)=x(t)$.

(2) Identify all local maxima and minima of $\tilde{x}(t)$.

(3) Find two envelopes $e_{\min }(t)$ and $e_{\max }(t)$ that interpolate through the local minima and maxima, respectively.

(4) Let $d(t)=\tilde{x}(t)-(1 / 2)\left(e_{\min }(t)+e_{\max }(t)\right)$ as the detail part of the signal.

(5) Let $\tilde{x}(t)=d(t)$ and go to step (2) and repeat until $d(t)$ becomes an IMF.

(6) Compute the residue $r(t)=x(t)-d(t)$ and go back to step (1) until the energy of the residue is below a threshold.

The extracted components satisfy the so-called monocomponent criteria, and the Hilbert transform can be applied to each IMF separately to obtain the phase information.

2.3. Multivariate EMD. In a lot of problems in engineering and physics, multichannel dynamics of the signals play an important role. However, these signals are processed channel-wise most of the time. Therefore, extension of EMD to multivariate signals is required for accurate datadriven time-frequency analysis of multichannel signals. Furthermore, joint analysis of multiple oscillatory components within a higher dimensional signal helps to circumvent the mode alignment problem [16]. The first complex extension of EMD was proposed by Tanaka and Mandic and employed the concept of analytical signal and subsequently applied standard EMD to analyze complex data [13]. An extension of EMD which operates fully in the complex domain was first proposed by Altaf et al. termed rotation-invariant EMD (RI-EMD) [14]. In the RI-EMD algorithm, the extrema of a complex signal are chosen to be the points where the angle of the derivative of the complex signal becomes zero and the signal envelopes are produced by using component-wise spline interpolation. An algorithm which gives more accurate values of the local mean is the bivariate EMD (BEMD) [15], where the envelopes corresponding to multiple directions in the complex plane are generated and then averaged to obtain the local mean. All of these methods are suitable for bivariate data analysis, but cannot extract time-frequency information for more than two signals simultaneously. Recently, extensions of EMD to trivariate and multivariate signals have been proposed [18]. The work proposed in this paper is based on the multivariate EMD and will be reviewed briefly in this section.

In real-valued EMD, the local mean is computed by taking an average of upper and lower envelopes, which in turn are obtained by interpolating between the local maxima and minima. However, for multivariate signals, the local maxima and minima may not be defined directly. To deal with this problem, multiple n-dimensional envelopes are generated by taking signal projections along different directions in $\mathrm{n}$-dimensional spaces. These envelopes are then averaged to obtain the local mean. This is a generalization of the concept employed in existing bivariate [15] and trivariate [17] extensions of EMD. The algorithm can be summarized as follows.

(1) Choose a suitable pointset for sampling on an $(n-1)$ sphere $((n-1)$ sphere resides in an $n$ dimensional Euclidean coordinate system).

(2) Calculate a projection, $\left.p^{\theta_{k}}(t)\right\}_{t=1}^{T}$, of the input signal $\mathbf{v}(\mathbf{t})_{t=1}^{T}$ along the direction vector, $\mathbf{x}^{\theta_{k}}$ for all $k$ giving $\left.p^{\theta_{k}}(t)\right\}_{k=1}^{K}$.

(3) Find the time instants $\mathbf{t}_{i}^{\theta_{k}}$ corresponding to the maxima of the set of projected signals $\left.p^{\theta_{k}}(t)\right\}_{k=1}^{K}$.

(4) Interpolate $\left[t_{i}^{\theta_{k}}, \mathbf{v}\left(t_{i}^{\theta_{k}}\right)\right]$ to obtain multivariate envelope curves $\left.\mathbf{e}^{\theta_{k}}(t)\right\}_{k=1}^{K}$.

(5) For a set of $K$ direction vectors, the mean of the envelope curves is calculated as $\mathbf{m}(t)=(1 / K) \sum_{k=1}^{K} \mathbf{e}^{\theta_{k}}(t)$.

(6) Extract the detail $d(t)$ using $d(t)=x(t)-m(t)$. If the detail fulfills the stoppage criterion for a multivariate IMF, apply the above procedure to $x(t)-d(t)$, otherwise apply it to $d(t)$.

The set of direction vectors can be treated as finding a uniform sampling scheme on an $n$ sphere, and in order to extract meaningful IMFs, the number of direction vectors, $K$, should be at least twice the number of data channels [18]. In this paper, the default value is $K=128$. The stoppage criterion for multivariate IMFs is similar to that proposed for univariate IMFs, the difference being that the condition for equality of the number of extrema and zero crossings is not imposed, as extrema cannot be properly defined for multivariate signals [32]. 


\section{Multivariate EMD-Based Multivariate Synchrony Measures}

3.1. Measures of Multivariate Synchronization. In the proposed work, we will develop measures of association based on bivariate phase synchrony in an attempt to capture multivariate synchronization effects. One measure of interest is $R^{2}$ like measure of association proposed by Robert and Escoufier [29], which is a multivariate generalization of Pearson correlation coefficient, defined as:

$$
R_{v}=\frac{\operatorname{tr}\left(\mathbf{R}_{\mathbf{x y}} \mathbf{R}_{\mathbf{y x}}\right)}{\sqrt{\operatorname{tr}\left(\mathbf{R}_{\mathbf{x x}}^{2}\right) \operatorname{tr}\left(\mathbf{R}_{\mathbf{y y}}^{2}\right)}},
$$

where $\mathbf{x}$ and $\mathbf{y}$ refer to groups of variables, $\mathbf{R}_{\mathbf{x y}}, \mathbf{R}_{\mathbf{y x}}, \mathbf{R}_{\mathbf{x x}}$, and $\mathbf{R}_{\mathbf{y y}}$ are the autocorrelation and cross-correlation matrices between the variables, and $R_{v}$ quantifies the association between the variables $x_{1}, x_{2}, \ldots, x_{q}$ and $y_{1}, y_{2}, \ldots, y_{p}$. This measure has been shown to be equivalent to a distance measure between normalized covariance matrices and is always between 0 and 1 . The numerator corresponds to a scalar product between positive semidefinite matrices, the denominator is the Frobenius matrix scalar product [33], and $R_{v}$ is equivalent to the cosine between the covariances of the two data matrices. The closer to 1 it is, the better is $\mathbf{y}$ as a substitute for $\mathbf{x}$. It has been shown that the major approaches within statistical multivariate data analysis, such as principal component analysis, canonical correlation, and multivariate regression, can all be brought into a common framework in which the $R_{v}$ coefficient is maximized subject to relevant constraints [29]. In the case of multivariate synchronization, the matrices $\mathbf{R}_{\mathbf{x y}}, \mathbf{R}_{\mathbf{y x}}, \mathbf{R}_{\mathbf{x x}}$, and $\mathbf{R}_{\mathbf{y y}}$ are formed by computing the pairwise bivariate phase synchrony across different groups of variables and within each group, respectively.

A second closely related measure that will be adapted for multivariate synchronization is the S-estimator [30], which quantifies the amount of synchronization within a group of oscillators using the eigenvalue spectrum of the correlation matrix:

$$
S=1+\frac{\sum_{i=1}^{N} \lambda_{i} \log \left(\lambda_{i}\right)}{\log (N)}
$$

where $\lambda_{i}$ s are the $N$-normalized eigenvalues. This measure is an information theoretic inspired measure since it is complement to the entropy of the normalized eigenvalues of the correlation matrix. The more dispersed the eigenspectrum is the higher the entropy would be. In this paper, this estimator will be applied to the bivariate synchronization matrix instead of the correlation matrix. If all of the oscillations in a group are completely synchronized, that is, the entries of the pairwise synchrony matrix are all equal to 1 , then all of the eigenvalues except one will be equal to zero, and the value of $S$ will be equal to 1 indicating perfect multivariate synchrony. This measure can quantify the amount of synchronization within a group of signals and thus is useful as a global complexity measure.
3.2. Proposed Approach. Let $N$ be the number of oscillators or channels in a system. The proposed multivariate phase synchronization measures can be computed from data as follows.

(1) Compute the $L$ IMFs for the $N$ oscillators, $x_{i}\left(t_{m}\right), m=$ $0,1, \ldots, M-1$ as described in Section 2 obtaining $y_{i}^{l}\left(t_{m}\right), i=1,2, \ldots, N, l=1,2, \ldots, L$ with $M$ being the number of time samples. The number of IMFs, $L$, is determined by the stopping criteria in MEMD.

For each IMF, $l$ :

(2) Compute the Hilbert transform, $\hat{y}_{i}^{l}(t)=H\left(y_{i}^{l}(t)\right)$, and obtain the phase as $\phi_{i}\left(t_{m}\right)=\phi_{i}^{l}\left(t_{m}\right)=$ $\arg \left[\arctan \left(\hat{y}\left(t_{m}\right) / y\left(t_{m}\right)\right)\right]$.

(3) Compute the pairwise synchrony (bivariate synchrony) between $i$ th and $j$ th oscillators, $\gamma_{i, j}$ :

$$
\gamma_{i, j}=\left|\frac{1}{M} \sum_{m=0}^{M-1} \exp \left(j\left(\phi_{i}\left(t_{m}\right)-\phi_{j}\left(t_{m}\right)\right)\right)\right| .
$$

(4) Form the bivariate phase synchrony matrix $R$ as

$$
R=\left[\begin{array}{cccc}
1 & \gamma_{1,2} & \ldots & \gamma_{1, N} \\
\gamma_{2,1} & 1 & \ddots & \gamma_{2, N} \\
\vdots & \vdots & \ddots & \vdots \\
\gamma_{N, 1} & \gamma_{N, 2} & \ldots & 1
\end{array}\right]
$$

(5) Using $R$, compute $S$ for the whole network by finding the normalized eigenvalues of $R$ and computing the expression given by (4).

(6) The measure $R_{v}$ quantifies the degree of association between two oscillator groups and can be computed for any groups of oscillators from the nextwork. For example, consider two oscillator groups, $\mathbf{x}$ and $\mathbf{y}$ formed by oscillators $\left\{1,2, \ldots, N^{\prime}\right\}$ and $\left\{N^{\prime}+1, \ldots, N\right\}$, respectively. $R_{v}$ between these two groups can be computed using (3) with matrices $\mathbf{R}_{\mathbf{x x}}, \mathbf{R}_{\mathbf{y y}}, \mathbf{R}_{\mathrm{xy}}$, and $\mathbf{R}_{\mathbf{y x}}$ computed as follows:

$$
\begin{aligned}
& \mathbf{R}_{\mathbf{x x}}=\left[\begin{array}{cccc}
1 & \gamma_{1,2} & \ldots & \gamma_{1, N^{\prime}} \\
\vdots & \ddots & \ddots & \gamma_{2, N^{\prime}} \\
\vdots & \ddots & \ddots & \vdots \\
\gamma_{N^{\prime}, 1} & \gamma_{N^{\prime}, 2} & \ddots & 1
\end{array}\right] \\
& \mathbf{R}_{\mathbf{y y}}=\left[\begin{array}{cccc}
1 & \gamma_{N^{\prime}+1, N^{\prime}+2} & \cdots & \gamma_{N^{\prime}+1, N} \\
\vdots & \ddots & \ddots & \gamma_{N^{\prime}+2, N} \\
\vdots & \ddots & \ddots & \vdots \\
& & \ddots & 1
\end{array}\right] \text {, }
\end{aligned}
$$




$$
\begin{array}{r}
\mathbf{R}_{\mathbf{x y}}=\left[\begin{array}{cccc}
\gamma_{1, N^{\prime}+1} & \gamma_{1, N^{\prime}+2} & \ldots & \gamma_{1, N} \\
\vdots & \ddots & \ddots & \gamma_{2, N} \\
\vdots & \ddots & \ddots & \vdots \\
\gamma_{N^{\prime}, N^{\prime}+1} & \gamma_{N^{\prime}, N^{\prime}+2} & \ddots & \gamma_{N^{\prime}, N}
\end{array}\right], \\
\mathbf{R}_{\mathbf{y x}}=\left[\begin{array}{cccc}
\gamma_{N^{\prime}+1,1} & \gamma_{N^{\prime}+1,2} & \ldots & \gamma_{N^{\prime}+1, N^{\prime}} \\
\vdots & \ddots & \ddots & \gamma_{N^{\prime}+2, N^{\prime}} \\
\vdots & \ddots & \ddots & \vdots \\
\gamma_{N, 1} & \gamma_{N, 2} & \ddots & \gamma_{N, N^{\prime}}
\end{array}\right] .
\end{array}
$$

The procedure described above can be extended to the case of computing synchrony across realizations instead of across time. This modification to step (4) would require the extraction of IMFs for each realization and computing the phase coherence by taking an average over realizations instead of time.

\section{Results}

4.1. Performance of MEMD-Based Phase Synchrony for Multicomponent Oscillators. In this example, we illustrate the usefulness of MEMD in quantifying phase synchrony across different frequency bands. If the analyzed signals are composed of multiple frequency components, phase synchrony can be observed in oscillators' different intrinsic time scales. Therefore, a decomposition based on the local characteristic time scales of the data is necessary to correctly detect the embedded nonstationary oscillations and their possible interactions [34]. In order to evaluate the performance of MEMD in decomposing nonstationary and multiple-frequency component signals into local time scales, a pair of unidirectionally coupled Van der Pol oscillators is considered:

$$
\begin{gathered}
\dot{x}=y, \\
\dot{y}=0.6 y\left(1-x^{2}\right)-x^{3}+C_{1} \sin 2 \pi f_{1} t+C_{2} \sin 2 \pi f_{2} t, \\
\dot{u}=v, \\
\dot{v}=0.2 v\left(1-u^{2}\right)-u^{3}+\epsilon(x-u),
\end{gathered}
$$

where $C_{1}=1, C_{2}=5, f_{1}=0.65$, and $f_{2}=0.25$. Subscripts $x y$ and $u v$ refer to the oscillators described by the variables $(x, y)$ and $(u, v)$, respectively. Coupling strength is fixed at $\epsilon=5$, and other coefficients are set such that both oscillators exhibit chaotic behavior for the uncoupled case [34]. The differential equations are numerically integrated using the Runge-Kutta method with a time step of $\Delta t=1 / 25$.

Since the $y$ component of the first signal includes two frequency components, phase synchrony between $x$ and $u$ should be observed at the IMFs with the mean frequencies, $f_{1}=0.65$ and $f_{2}=0.25 \mathrm{~Hz} .100$ simulations of the model is generated with additive white Gaussian noise at a SNR value of $10 \mathrm{~dB}$. The largest two synchrony values with mean and standard deviations, $0.5109 \pm 0.0427$ and $0.9482 \pm 0.0361$, are obtained at the 5th and 6th IMFs which oscillate at the mean frequencies 0.65 and $0.25 \mathrm{~Hz}$, respectively. This result is consistent with the model where the synchrony provided by the 6 th IMF is greater than the one provided by the 5 th IMF, since $C_{2}$ is greater than $C_{1}$. A sample decomposition of $x, y, u$ and $v$ into their IMFs using MEMD is given in Figure 1 .

4.2. Rossler Oscillator Model. In the remainder of the paper, in order to evaluate the performance of the proposed multivariate measures, a well-known model of nonlinear oscillators, called Rossler oscillators, is used. These chaotic oscillators, investigated by $[2,35]$, form a system that is known to have characteristic phase synchronization properties and to exhibit clusters of phase synchronization depending on the coupling strengths within the system. The model consists of a network of multivariate time series coupled in a way to form synchronization clusters of different size as well as desynchronized oscillators. The networks considered in this paper consist of $N=6$ Rossler oscillators which are coupled diffusively via their $z$-components:

$$
\begin{aligned}
& \dot{x}_{j}=10\left(y_{j}-x_{j}\right), \\
& \dot{y}_{j}=28 x_{j}-y_{j}-x_{j} z_{j}, \\
& \dot{z}_{j}=-\frac{8}{3 z_{j}}+x_{j} y_{j}+\sum_{i=1}^{N} \epsilon_{i j}\left(z_{i}-z_{j}\right) .
\end{aligned}
$$

The coupling coefficients, $\epsilon_{i j}$, are chosen from the interval $[0,1]$ to construct different networks. The differential equations are numerically integrated using the Runge-Kutta method with a time step of $\Delta t=1 / 25 \mathrm{sec}$ corresponding to a sampling frequency of $25 \mathrm{~Hz}$, where the initial conditions are randomly chosen from the interval, $[0,100]$. The first 2500 samples are discarded to eliminate the initial transients.

4.3. Comparison of Direct Application of Hilbert Transform with Multivariate EMD. In order to illustrate the advantage of using multivariate EMD as a preprocessing tool over the direct application of the Hilbert transform in estimating the time-varying phase synchrony, the Rossler network in (9) is simulated using 1300 samples (see Figure 4(a)), where the coupling strengths, $\epsilon_{1,2}=\epsilon_{2,1}=\epsilon_{1,3}=\epsilon_{3,1}=$ $\epsilon_{2,3}=\epsilon_{3,2}=1, \epsilon_{4,5}=\epsilon_{5,4}=\epsilon_{4,6}=\epsilon_{6,4}=\epsilon_{5,6}=$ $\epsilon_{6,5}=1$ and all other coupling strengths are set to zero, such that the network consists of two strongly synchronized clusters with no between-cluster coupling. The first cluster is shown in green and the second cluster is shown in yellow in Figure 4(a). In this example, three $S$ values representing the phase synchrony within the clusters (green, yellow, and the whole network) and the $R_{v}$ value representing the synchrony between the clusters are computed. In the absence of noise, when the phase synchrony analysis is performed for the $z$-components of the Rossler oscillators using the Hilbert transform, $S$ and $R_{v}$ values are computed as $S_{g}=1$ 

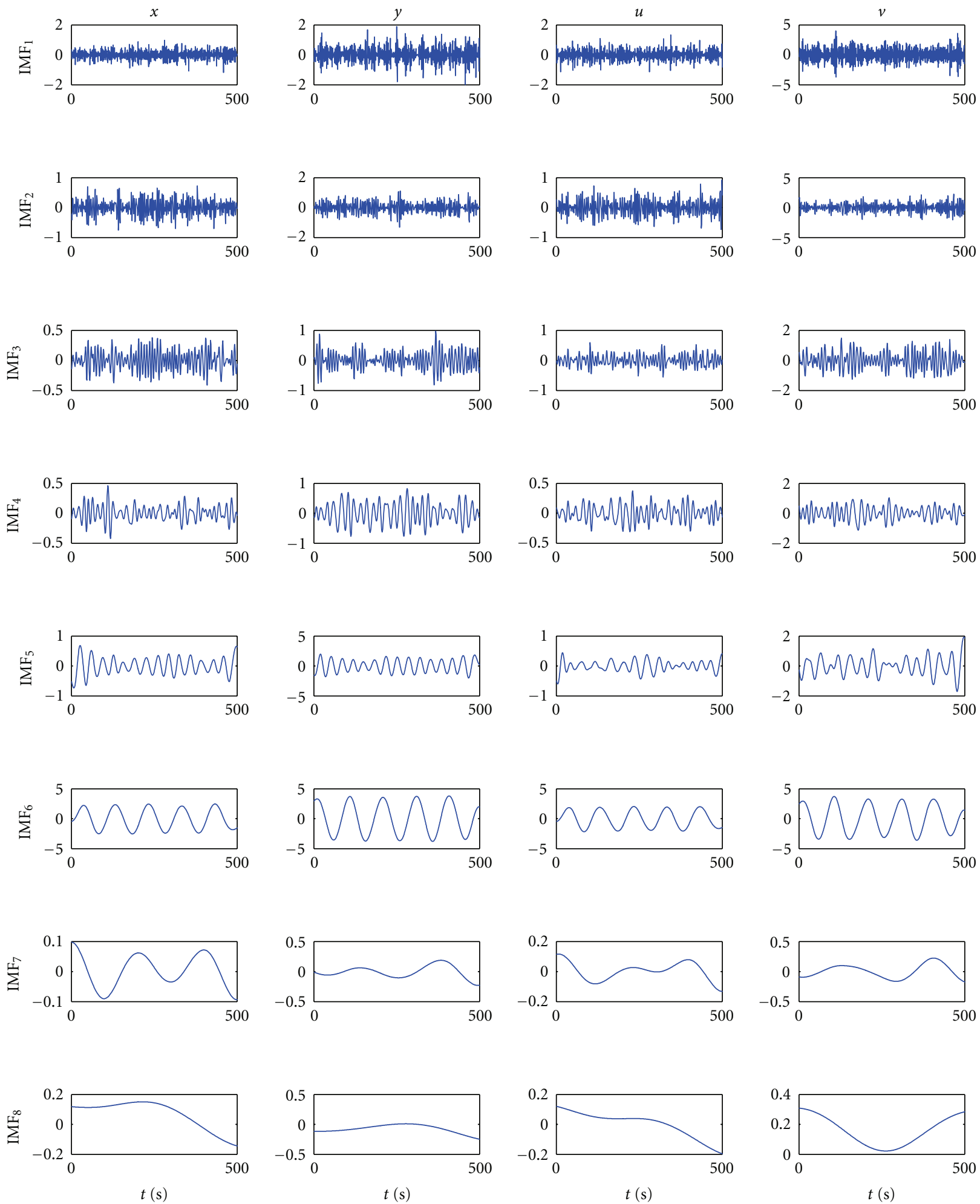

FIGURE 1: Decomposition of $x, y, u$, and $v$ in (8) into the IMFs by MEMD: 5th and 6th IMFs which oscillate at mean frequencies 0.65 and $0.25 \mathrm{~Hz}$, respectively, provide the largest synchrony values between $x$ and $u$. 


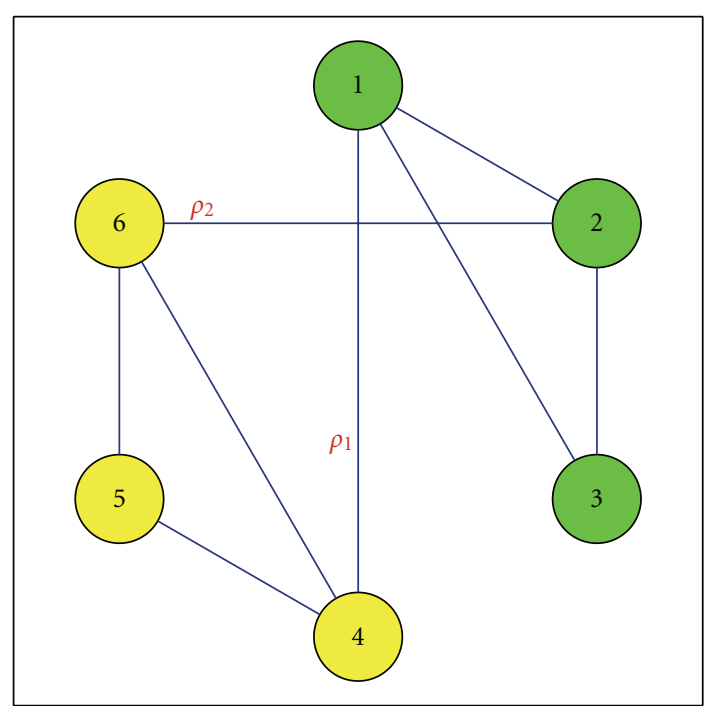

FIGURE 2: Rossler network for evaluating the dependency of the multivariate synchrony measures on the coupling strengths. The coupling strengths $\rho_{1}$ and $\rho_{2}$ are increased from 0 to 1 in steps of 0.2 .

( $S$ value computed for the first cluster), $S_{y}=1$ (second cluster), $S_{T}=0.6175$ (network consisting of all 6 oscillators), and $R_{v}=0.007$. These values agree with our intuition, since the $S$-estimator is proportional to the amount of withincluster synchronization and $R_{v}$-estimator is proportional to the amount of between-cluster synchronization. However, Hilbert transform is actually a filter with unit gain at every frequency [2], so that the whole range of frequencies is taken into account to define the instantaneous phase. Therefore, if the signal is broadband it is necessary to prefilter it in the frequency band of interest before applying the Hilbert transform, in order to get an accurate estimate of the phase (e.g., $[8,9])$. Therefore, instead of bandpass filtering the oscillators, multivariate EMD will be employed, and for the Rossler networks, the IMFs with the highest energies will be used for the synchrony analysis, since these networks consist of monocomponent oscillators.

To show the advantage of using multivariate EMD, 50 simulations of the Rossler network are performed with additive white Gaussian noise at a SNR value of $0 \mathrm{~dB}$. When the Hilbert transform is used directly, the mean values and the standard deviations of the $S$ and $R_{v}$ estimators are computed as $S_{g}=0.1651 \pm 0.0162, S_{y}=0.1617 \pm 0.0172$, $S_{T}=0.1069 \pm 0.0095$, and $R_{v}=0.1413 \pm 0.0243$. These results are not close to the ideal values of $S$ and $R_{v}$ estimators given above. This is caused by the broadband nature of the noise and the fact that the Hilbert transform is actually a filter with unit gain at every frequency. However, when multivariate EMD is used and the IMFs with the highest energies are extracted from each oscillator, the mean values and the standard deviations (mean \pm std) of the $S$ and $R_{v}$ estimators are computed as $S_{g}=0.7888 \pm 0.0377, S_{y}=0.7771 \pm 0.0349$, $S_{T}=0.5144 \pm 0.0362$, and $R_{v}=0.1214 \pm 0.0653$. These results are much closer to the ideal values of $S$ and $R_{v}$, which shows the advantage of using multivariate EMD as a preprocessing tool for phase synchrony analysis.
4.4. Performance of Multivariate Synchrony Measures for Multivariate EMD. In this example, the dependency of the multivariate synchrony measures on the coupling strengths in a Rossler network is evaluated using 500 samples. The network in Figure 2 is formed, and the coupling strengths $\rho_{1}=\epsilon_{1,4}=\epsilon_{4,1}$ and $\rho_{2}=\epsilon_{2,6}=\epsilon_{6,2}$ are increased from 0 to 1 in steps of 0.2 , with $\epsilon_{1,2}=\epsilon_{2,1}=\epsilon_{1,3}=\epsilon_{3,1}=\epsilon_{2,3}=\epsilon_{3,2}=1$, $\epsilon_{4,5}=\epsilon_{5,4}=\epsilon_{4,6}=\epsilon_{6,4}=\epsilon_{5,6}=\epsilon_{6,5}=1$, and all other coupling strengths set to zero.

Figure 3 shows the dependency of the $S_{g}, S_{y}, S_{T}$, and $R_{v}$ on the coupling strengths $\rho_{1}$ and $\rho_{2}$, in the absence of noise. When both $\rho_{1}$ and $\rho_{2}$ are equal to zero, $S_{g}$ and $S_{y}$ have the highest values, which is equivalent to the network in Figure 4(a). In this case, there are two completely separate clusters, and each cluster has the maximum phase synchrony, with no between-cluster synchrony. This result is expected, since the $S$ values represent the within-cluster phase synchrony and increasing the coupling coefficients $\rho_{1}$ and $\rho_{2}$ synchronizes the two of the oscillators from each cluster to the other two oscillators in the other cluster, which destroys the within-cluster phase synchrony. Thus, maximum within-cluster synchrony is achieved when $\rho_{1}=0$ and $\rho_{2}=0$ and increasing either or both $\rho_{1}$ and $\rho_{2}$ results in the reduced within-cluster phase synchrony values, shown by Figures 3(a) and 3(b).

$S_{T}$, which shows the within-cluster synchrony for the whole network, has the maximum value when $\rho_{1}$ and $\rho_{2}$ are both equal to 1 . This is also an expected result since these two coupling strengths try to synchronize the two clusters with each other. Reduction in either or both of $\rho_{1}$ and $\rho_{2}$ results in a reduced $S_{T}$ value, which is shown in Figure 3(c).

Figure 3(d) shows that $R_{v}$, which represents the betweencluster synchrony, is directly proportional to $\rho_{1}$ and $\rho_{2}$. An increase in only one of the coupling strengths is not enough to increase $R_{v}$. However, when both of these coupling strengths increase, $R_{v}$ also increases and reaches its maximum value when $\rho_{1}=1$ and $\rho_{2}=1$. This is an expected result since both $\rho_{1}$ and $\rho_{2}$ are responsible for the increased between-cluster synchrony. Moreover, by looking at Figures $3(\mathrm{c})$ and $3(\mathrm{~d})$, one can say that there is a strong positive correlation between $S_{T}$ and $R_{v}$. The reason for this is that $R_{v}$ represents the between-cluster synchrony and $S_{T}$ represents the synchrony, of the whole network, both of which increase with the increasing coupling strengths, $\rho_{1}$ and $\rho_{2}$.

In order to evaluate the performance of the $S$ - and $R_{v^{-}}$ estimators in estimating the within-cluster and betweencluster synchrony in detail, 12 different Rossler networks, shown in Figure 4, consisting of 6 oscillators are generated. Each connection represents two symmetric coupling strengths, equal to 1 , between two oscillators. The $z$ components of the oscillators are preprocessed by the multivariate EMD, and the IMFs with the highest energies are extracted from each oscillator. The IMFs with the highest energies correspond to the same mode for all 6 oscillators. For each network, 50 simulations are performed with additive white Gaussian noise at a SNR value of $0 \mathrm{~dB}$.

Table 1 shows the mean and standard deviation values for all 12 networks. Networks 1 and 5 have the largest $S_{g}$ and $S_{y}$ values, which indicates that the within-cluster 


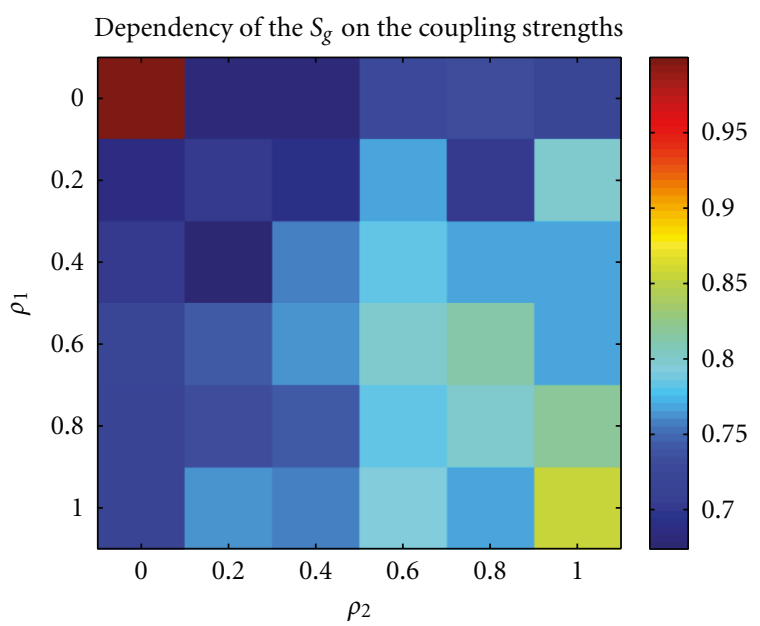

(a) $S_{g}$

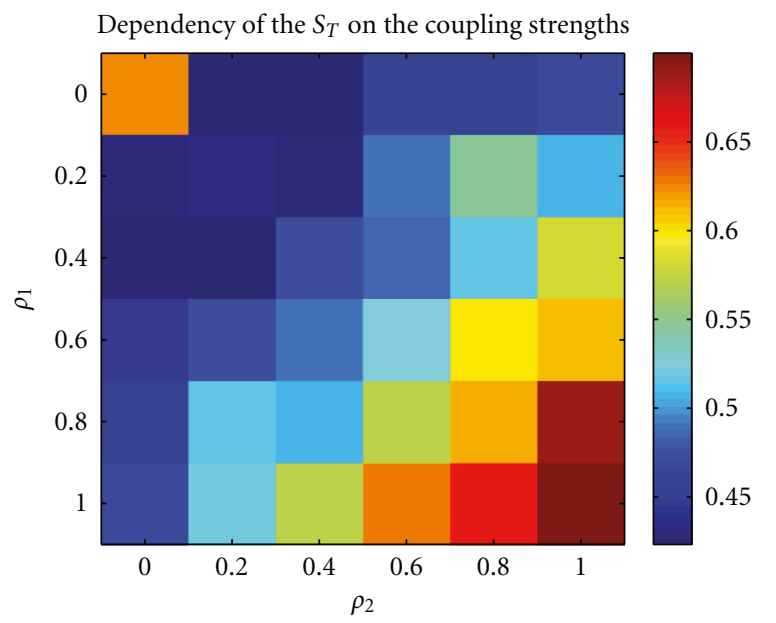

(c) $S_{T}$

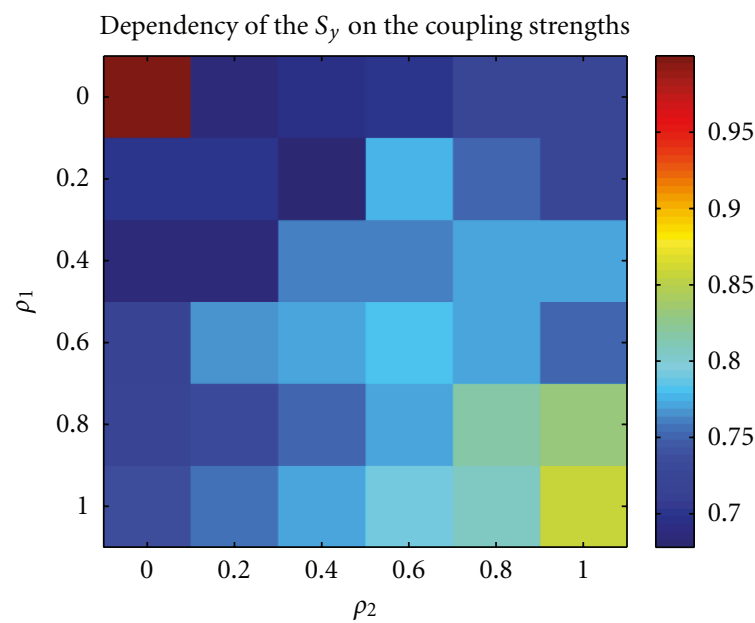

(b) $S_{y}$

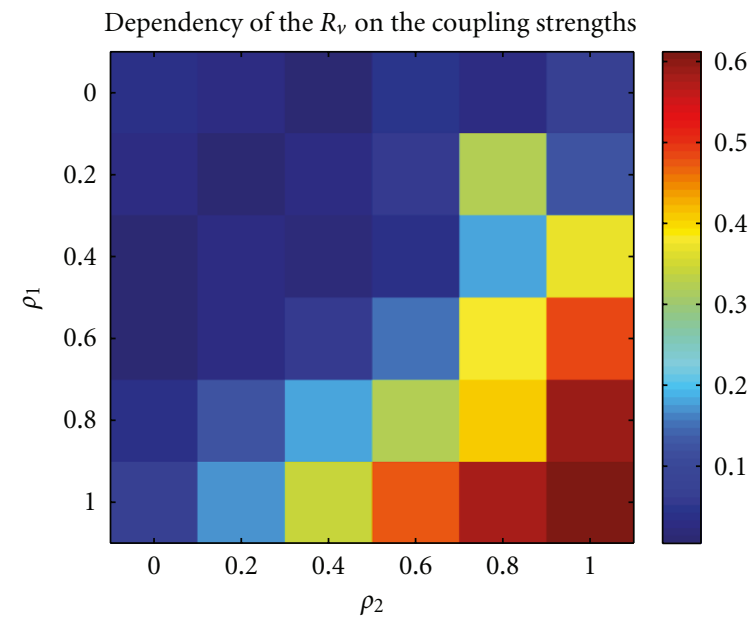

(d) $R$

FIGURE 3: Dependency of the multivariate synchrony measures, $S_{g}, S_{y}, S_{T}$, and $R_{v}$, on the coupling strengths $\rho_{1}$ and $\rho_{2}$.

TABLE 1: Means and standard deviations of $R_{v}, S_{g}, S_{y}$, and $S_{T}$ for the networks in Figure 4.

\begin{tabular}{lcccc}
\hline & $R_{v}$ & $S_{g}$ & $S_{y}$ & $S_{T}$ \\
\hline Network 1 & $0.2131 \pm 0.1786$ & $0.7670 \pm 0.0631$ & $0.7827 \pm 0.0665$ & $0.5390 \pm 0.0668$ \\
Network 2 & $0.1306 \pm 0.0961$ & $0.3605 \pm 0.2239$ & $0.4490 \pm 0.2556$ & $0.2881 \pm 0.1183$ \\
Network 3 & $0.1476 \pm 0.1364$ & $0.5826 \pm 0.1342$ & $0.5641 \pm 0.1230$ & $0.4038 \pm 0.0763$ \\
Network 4 & $0.6160 \pm 0.2165$ & $0.6398 \pm 0.1445$ & $0.6644 \pm 0.1375$ & $0.5947 \pm 0.1487$ \\
Network 5 & $0.9380 \pm 0.0266$ & $0.7687 \pm 0.0527$ & $0.7631 \pm 0.0594$ & $0.7956 \pm 0.0491$ \\
Network 6 & $0.7539 \pm 0.3110$ & $0.4130 \pm 0.2489$ & $0.4153 \pm 0.2409$ & $0.5047 \pm 0.2446$ \\
Network 7 & $0.6913 \pm 0.2354$ & $0.4735 \pm 0.1945$ & $0.4876 \pm 0.2016$ & $0.5175 \pm 0.1884$ \\
Network 8 & $0.2350 \pm 0.1687$ & $0.3046 \pm 0.2004$ & $0.2944 \pm 0.1997$ & $0.2624 \pm 0.1537$ \\
Network 9 & $0.4376 \pm 0.1319$ & $0.4070 \pm 0.0881$ & $0.4192 \pm 0.0959$ & $0.4164 \pm 0.0671$ \\
Network 10 & $0.7774 \pm 0.0695$ & $0.4056 \pm 0.0946$ & $0.3941 \pm 0.0976$ & $0.5305 \pm 0.0761$ \\
Network 11 & $0.3479 \pm 0.1374$ & $0.4043 \pm 0.0879$ & $0.4046 \pm 0.0818$ & $0.3996 \pm 0.0566$ \\
Network 12 & $0.4196 \pm 0.1361$ & $0.4092 \pm 0.0836$ & $0.1777 \pm 0.0912$ & $0.3455 \pm 0.0629$ \\
\hline
\end{tabular}




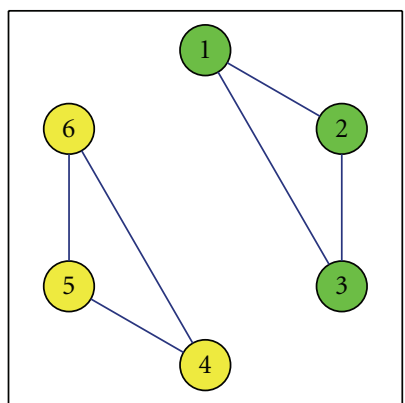

(a) Network 1

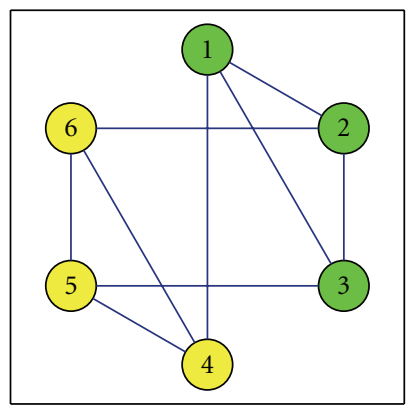

(e) Network 5

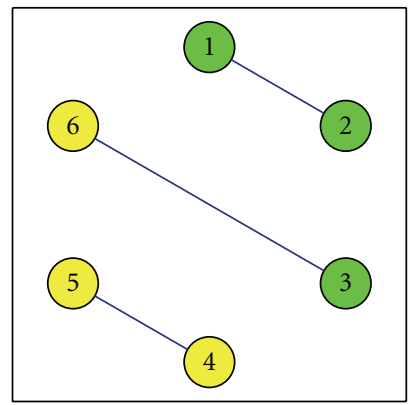

(i) Network 9

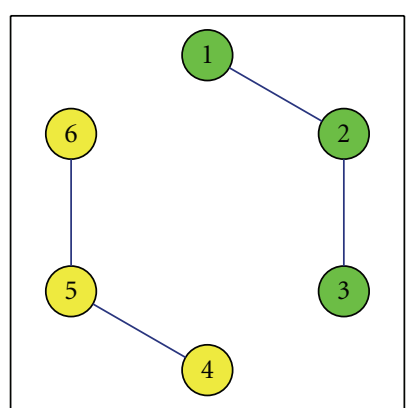

(b) Network 2

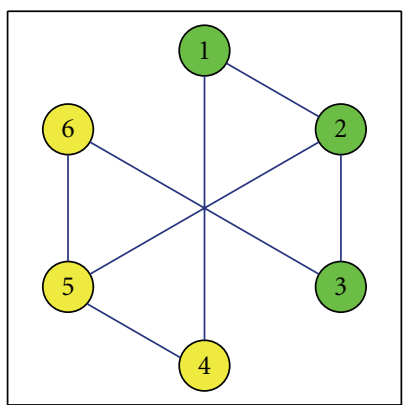

(f) Network 6

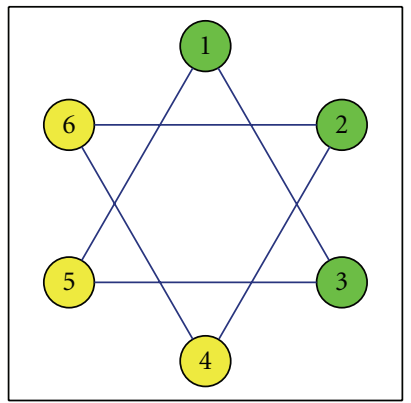

(j) Network 10

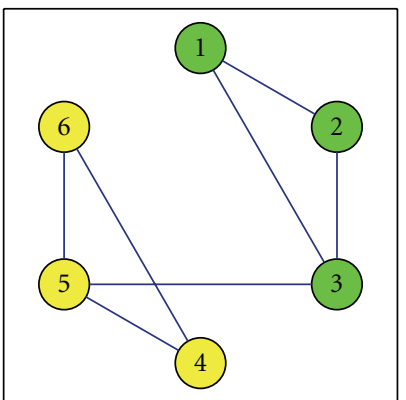

(c) Network 3

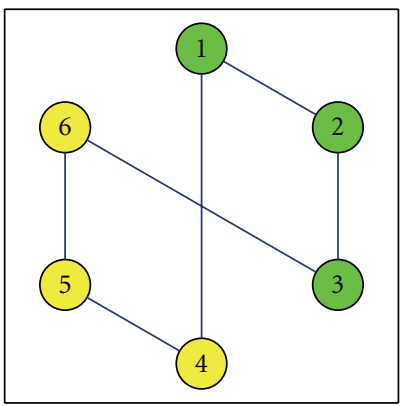

(g) Network 7

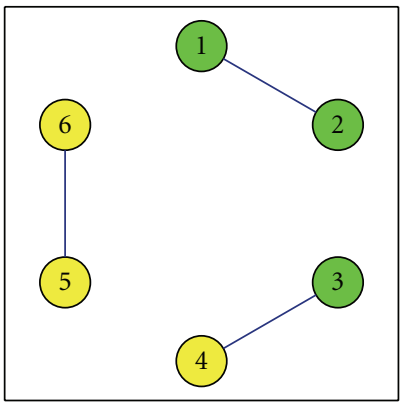

(k) Network 11

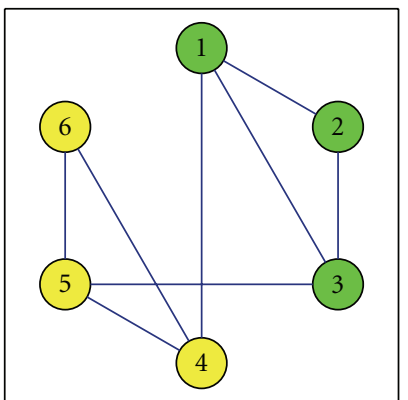

(d) Network 4

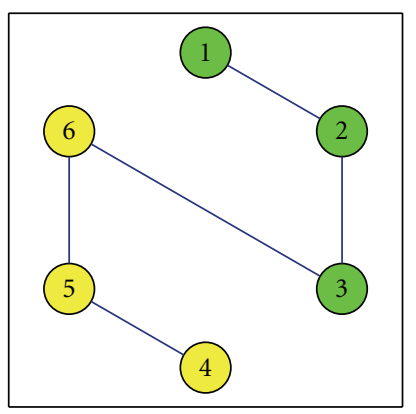

(h) Network 8

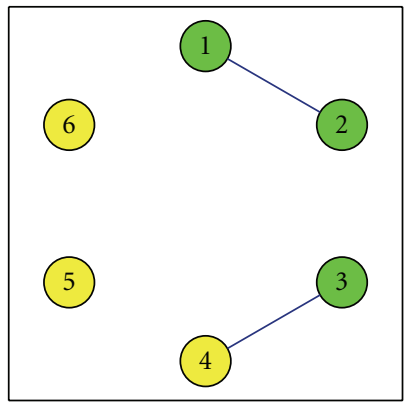

(1) Network 12

FIGURE 4: 12 different Rossler networks for evaluating the performance of the $S$ - and $R_{v}$-estimators in estimating the within-cluster and between-cluster synchrony. Each node represents an oscillator, and each connection represents the two symmetric coupling strengths, which are equal to 1 , between two oscillators.

phase synchrony is very strong for these networks. Strong within-cluster synchrony, or high $S$ value, is usually obtained when all possible within-cluster connections exist and the between-cluster connections are either very strong or nonexistent. Networks 1 and 5 satisfy these conditions with network 1 having no between-cluster connections and network 5 having strong connections between the two clusters. On the other hand, networks 3 and 4 have all possible within-cluster connections, but they have smaller $S_{g}$ and $S_{y}$ values compared to network 5, since the small number of between-cluster connections are not adequate to synchronize the two clusters and are also disruptive to the within-cluster synchrony.

The largest $R_{v}$ value, or between-cluster synchrony, is observed for network 5 which results from the three connections between the two clusters, forcing the two clusters to be highly synchronized with each other. Networks 6 and 10 also have a large number of connections between the two clusters but they lack some of the within-cluster connections, which results in reduced $R_{v}$ values for these networks. Network 6 has a larger $R_{v}$ value compared to network 7 but has smaller $S$ values, since there are 3 connections between the clusters. Networks $8,9,11$, and 12 all have small $R_{v}$ and $S$ values, since the within-cluster and between-cluster synchronies are both weak due to the lack of connectivity in these networks.

$S_{T}$, on the other hand, measures the within-cluster synchronization when all six oscillators are assumed to form a big cluster. Network 5 has the largest $S_{T}$ value, because there is one big cluster which is formed by multiple smaller clusters with strong within-cluster connectivity. Since the connectivity in the whole network is strong, the eigenvalues of the synchrony matrix tend to be better concentrated, which results in a low entropy value and a high $S_{T}$ value. On the other hand, networks 2 and 8 have small $S_{T}$ values, since the within-network connectivity is not strong. 
4.5. Significance Testing for the Multivariate Synchrony Measures. Determining the statistical significance involves hypothesis testing and requires the formation of a null hypothesis. In some cases, it may be possible to derive analytically the distribution of the given measure under a given null hypothesis. However, in the case of the multivariate synchronization measures, this proves to be a very difficult problem, therefore this distribution is estimated by direct Monte Carlo simulations. For this purpose, an ensemble of surrogate data sets are generated [36]. The surrogate data set is generated by first computing the Fourier transform of the data and then randomizing the phase. Finally, the inverse Fourier transform is taken to obtain the surrogate data which has the same power spectrum and autocorrelation function as the original data. This operation preserves the amplitude relationships while randomizing the phase dependencies. For each surrogate data set, the MEMD is applied, the IMFs are extracted, and the corresponding multivariate measures are computed. From this ensemble of statistics, the distribution is approximated. A robust way to define significance would be directly in terms of the $P$-values with rank statistics. For example, if the observed time series has a $R_{v}$ or $S$ value, that is in the lower one percentile of all the surrogate statistics, then a $P$-value of $P=.01$ could be quoted. For the networks in Figure 3, 100 surrogate data sets are formed, and all multivariate synchrony measures, $R_{v}, S_{g}, S_{y}$, and $S_{T}$, are found to be significant at $P=.05$. The surrogate testing demonstrates that our simulation results are not likely to occur by chance.

4.6. Application to EEG Data. The proposed multivariate phase synchronization measures in conjunction with multivariate EMD were applied to a set of EEG data containing the error-related negativity (ERN). The ERN is a brain potential response that occurs following performance errors in a speeded reaction time task [37]. Previous work [38] indicates that there is increased phase synchrony associated with ERN for the theta frequency band $(4-7 \mathrm{~Hz})$ and ERN time window $(25-75 \mathrm{~ms})$ between frontal and central electrodes versus central and parietal. EEG data from 63channels was collected in accordance with the 10/20 system on a Neuroscan Synamps2 system (Neuroscan, Inc.) (The authors would like to acknowledge Dr. Edward Bernat from Florida State University for sharing his EEG data with us). A speeded-response flanker task was employed, and responselocked averages were computed for each subject.

In this paper, we analyzed data from 11 subjects corresponding to the error responses from five electrodes corresponding to the areas of interest, that is, two frontal electrodes (F3 and F4), one central electrode (FCz), and two parietal electrodes (CP3 and CP4). All five electrodes were first transformed using multivariate EMD, and the IMF closest to the theta frequency band was selected. The five IMFs were used to estimate phase and compute the pairwise phase synchrony. After the $5 \times 5$ bivariate synchronization matrix was formed, we computed $S$ and $R_{v}$ values considering two groups of electrodes, F3, F4, and FCz and $\mathrm{CP} 3, \mathrm{CP} 4$, and FCz.
For all 11 subjects, $S$ value of the electrode group, F3F4-FCz, is larger than the $S$ value of the group, CP3-CP4FCz. A Wilcoxon rank sum test is used at $\% 5$ significance level to test the null hypothesis that the $S$ values of the group $\mathrm{F} 3-\mathrm{F} 4-\mathrm{FCz}$ and the $S$ values of the group CP3-CP4$\mathrm{FCz}$ are independent samples from identical distributions with equal medians, against the alternative that they do not have equal medians. The $P$-value provided by the test is .0215 , which is less than .05 . Thus, the null hypothesis is rejected at $5 \%$ significance level, which shows that the $S$ values of the group $\mathrm{F} 3-\mathrm{F} 4-\mathrm{FCz}$ are significantly larger than the $S$ values of the group $\mathrm{CP} 3-\mathrm{CP} 4-\mathrm{FCz}$. This result indicates that the frontal (F3 and F4) electrodes are more strongly coupled to the central electrode $(\mathrm{FCz})$, compared to the coupling between the parietal (CP3 and $\mathrm{CP} 4)$ and central electrodes, which is shown by the significantly larger within-cluster synchrony values. Moreover, $R_{v}$ value between the central-frontal and $R_{v}$ value between the central-parietal electrode groups are computed. $R_{v}$ value between the centralfrontal electrodes is larger for 10 subjects out of 11 . Using the Wilcoxon rank sum test, the null hypothesis is rejected with $P$-value $=.0181$, which demonstrates that the synchrony between the frontal (F3 and F4) electrodes and central electrode $(\mathrm{FCz})$ is larger compared to the synchrony between the parietal (CP3 and $\mathrm{CP} 4)$ and central electrodes, which is shown by the significantly larger between-cluster synchrony. These results are consistent with the previous work in [38].

\section{Conclusions}

In this paper, a new approach for quantifying multivariate phase synchronization within a group of oscillators as well as between groups has been introduced. The proposed approach is based on the application of multivariate empirical mode decomposition for extracting time- and frequencydependent phase information, and adapting measures of correlation from statistics to multivariate analysis. The proposed approach offers improvements over existing methods in two ways. First, the MEMD is data driven in the sense that it extracts modes or frequencies that are common to all of the signals under consideration, thus eliminating the need for arbitrarily chosen bandpass filters as in the case of the Hilbert and the wavelet transforms. The timevarying phase information extracted through MEMD reflects the underlying nature of the signals and is more efficient compared to standard transform based approaches where each frequency is considered regardless of its importance. Second, the proposed approach extends the current state of the art-phase synchrony analysis from quantifying bivariate relationships to multivariate ones. This shift from pairwise bivariate synchrony analysis to multivariate analysis within and across groups offers advantages especially for complex system analysis such as the brain, where the bivariate relationships do not always reflect the underlying network structure. The multivariate synchrony measures, such as the ones introduced in this paper, are a step in the right direction for understanding the network dynamics. 
Future work will focus on the extension of the proposed measures using different multivariate analysis techniques such as cluster analysis and canonical correlation to obtain a more detailed understanding of the synchronization networks as well as the application of these measures to a large number of signals, as is the case with EEG. In the current application to EEG data, we focused on a group of electrodes with known synchronization patterns. It would be valuable to apply the proposed approach to the whole set of electrodes and discover the underlying synchronization clusters through a combination of eigenvalue decomposition and measures of association and complexity, for example, $R_{v}$ and $S$.

\section{Acknowledgment}

This paper was in part supported by grants from the National Science Foundation under CCF-0728984 and CAREER CCF0746971.

\section{References}

[1] E. Pereda, R. Q. Quiroga, and J. Bhattacharya, "Nonlinear multivariate analysis of neurophysiological signals," Progress in Neurobiology, vol. 77, no. 1-2, pp. 1-37, 2005.

[2] M. G. Rosenblum, A. S. Pikovsky, and J. Kurths, "Phase synchronization of chaotic oscillators," Physical Review Letters, vol. 76, no. 11, pp. 1804-1807, 1996.

[3] P. Tass, M. G. Rosenblum, J. Weule et al., "Detection of n:m phase locking from noisy data: application to magnetoencephalography," Physical Review Letters, vol. 81, no. 15, pp. 3291-3294, 1998.

[4] J. P. Lachaux, A. Lutz, D. Rudrauf et al., "Estimating the time-course of coherence between single-trial brain signals: an introduction to wavelet coherence," Neurophysiologie Clinique, vol. 32, no. 3, pp. 157-174, 2002.

[5] S. Mallat, A Wavelet Tour of Signal Processing, Academic Press, New York, NY, USA, 1999.

[6] M. Le Van Quyen, J. Foucher, J. P. Lachaux et al., "Comparison of Hilbert transform and wavelet methods for the analysis of neuronal synchrony," Journal of Neuroscience Methods, vol. 111, no. 2, pp. 83-98, 2001.

[7] L. Angelini, M. De Tommaso, M. Guido et al., "Steadystate visual evoked potentials and phase synchronization in migraine patients," Physical Review Letters, vol. 93, no. 3, Article ID 038103, 2004.

[8] J. Bhattacharya, "Reduced degree of long-range phase synchrony in pathological human brain," Acta Neurobiologiae Experimentalis, vol. 61, no. 4, pp. 309-318, 2001.

[9] M. Koskinen, T. Seppänen, J. Tuukkanen, A. Yli-Hankala, and V. Jäntti, "Propofol anesthesia induces phase synchronization changes in EEG," Clinical Neurophysiology, vol. 112, no. 2, pp. 386-392, 2001.

[10] T. M. Rutkowski, D. P. Mandic, A. Cichocki, and A. W. Przybyszewski, "EMD approach to multichannel EEG datathe amplitude and phase components clustering analysis," Journal of Circuits, Systems and Computers, vol. 19, no. 1, pp. 215-229, 2010.

[11] C. M. Sweeney-Reed and S. J. Nasuto, "A novel approach to the detection of synchronisation in EEG based on empirical mode decomposition," Journal of Computational Neuroscience, vol. 23, no. 1, pp. 79-111, 2007.
[12] D. Looney, C. Park, P. Kidmose, M. Ungstrup, and D. P. Mandic, "Measuring phase synchrony using complex extensions of EMD," in Proceedings of the IEEE/SP 15th Workshop on Statistical Signal Processing (SSP '09), pp. 49-52, August 2009.

[13] T. Tanaka and D. P. Mandic, "Complex empirical mode decomposition,” IEEE Signal Processing Letters, vol. 14, no. 2, pp. 101-104, 2007.

[14] M. Bin Altaf, T. Gautama, T. Tanaka, and D. P. Mandic, "Rotation invariant complex empirical mode decomposition," in Proceedings of the IEEE International Conference on Acoustics, Speech and Signal Processing (ICASSP '07), vol. 3, pp. 10091012, April 2007.

[15] G. Rilling, P. Flandrin, P. Goncalves, and J. M. Lilly, "Bivariate empirical mode decomposition," IEEE Signal Processing Letters, vol. 14, no. 12, pp. 936-939, 2007.

[16] D. Looney and D. P. Mandic, "Multiscale image fusion using complex extensions of EMD," IEEE Transactions on Signal Processing, vol. 57, no. 4, pp. 1626-1630, 2009.

[17] N. Rehman and D. Mandic, "Empirical mode decomposition for trivariate signals," IEEE Transactions on Signal Processing, vol. 58, no. 3, pp. 1059-1068, 2010.

[18] N. Rehman and D. P. Mandic, "Multivariate empirical mode decomposition," Proceedings of the Royal Society A: Mathematical, Physical and Engineering Sciences, vol. 466, no. 2117, pp. 1291-1302, 2010.

[19] B. Schelter, M. Winterhalder, R. Dahlhaus, J. Kurths, and J. Timmer, "Partial phase synchronization for multivariate synchronizing systems," Physical Review Letters, vol. 96, no. 20, Article ID 208103, 2006.

[20] M. E. J. Newman, "Finding community structure in networks using the eigenvectors of matrices," Physical Review E Statistical, Nonlinear, and Soft Matter Physics, vol. 74, no. 3, Article ID 036104, 2006.

[21] M. E. J. Newman, "Modularity and community structure in networks," Proceedings of the National Academy of Sciences of the United States of America, vol. 103, no. 23, pp. 8577-8582, 2006.

[22] C. Allefeld and J. Kurths, "An approach to multivariate phase synchronization analysis and its application to event-related potentials," International Journal of Bifurcation and Chaos, vol. 14, no. 2, pp. 417-426, 2004.

[23] C. Allefeld, M. Müller, and J. Kurths, "Eigenvalue decomposition as a generalized synchronization cluster analysis," International Journal of Bifurcation and Chaos, vol. 17, no. 10, pp. 3493-3497, 2007.

[24] C. Allefeld, S. Frisch, and M. Schlesewsky, "Detection of early cognitive processing by event-related phase synchronization analysis," NeuroReport, vol. 16, no. 1, pp. 13-16, 2005.

[25] C. Allefeld and J. Kurths, "Multivariate phase synchronization analysis of EEG data," IEICE Transactions on Fundamentals of Electronics, Communications and Computer Sciences, vol. 86, no. 9, pp. 2218-2221, 2003.

[26] C. Allefeld and S. Bialonski, "Detecting synchronization clusters in multivariate time series via coarse-graining of Markov chains," Physical Review E, vol. 76, no. 6, Article ID 066207, pp. 66207-66215, 2007.

[27] C. W. J. Granger, "Investigating causal relations by econometric models and cross-spectral methods," Econometrica, vol. 37, no. 3, pp. 424-438, 1969.

[28] L. A. Baccalá and K. Sameshima, "Partial directed coherence: a new concept in neural structure determination," Biological Cybernetics, vol. 84, no. 6, pp. 463-474, 2001. 
[29] P. Robert and Y. Escoufier, "A unifying tool for linear multivariate statistical methods: the RV-coefficient," Applied Statistics, vol. 25, no. 3, pp. 257-265, 1976.

[30] K. Oshima, C. Carmeli, and M. Hasler, "State change detection using multivariate synchronization measure from physiological signals," Journal of Signal Processing, vol. 10, no. 4, pp. 223226, 2006.

[31] N. E. Huang, Z. Shen, S. R. Long et al., "The empirical mode decomposition and the Hubert spectrum for nonlinear and non-stationary time series analysis," Proceedings of the Royal Society A: Mathematical, Physical and Engineering Sciences, vol. 454, no. 1971, pp. 903-995, 1998.

[32] D. Mandic and V. Goh, Complex Valued Nonlinear Adaptive Filters: Noncircularity, Widely Linear and Neural Models, Wiley Publishing, 2009.

[33] R. Horn and C. Johnson, Matrix Analysis, Cambridge University Press, Cambridge, UK, 1990.

[34] M. Chavez, C. Adam, V. Navarro, S. Boccaletti, and J. Martinerie, "On the intrinsic time scales involved in synchronization: a data-driven approach," Chaos, vol. 15, no. 2, Article ID 023904, pp. 1-11, 2005.

[35] G. V. Osipov, A. S. Pikovsky, M. G. Rosenblum, and J. Kurths, "Phase synchronization effects in a lattice of nonidentical Rössler oscillators," Physical Review E, vol. 55, no. 3, pp. 2353 2361, 1997.

[36] D. Prichard and J. Theiler, "Generating surrogate data for time series with several simultaneously measured variables," Physical Review Letters, vol. 73, no. 7, pp. 951-954, 1994.

[37] J. R. Hall, E. M. Bernat, and C. J. Patrick, "Externalizing psychopathology and the error-related negativity," Psychological Science, vol. 18, no. 4, pp. 326-333, 2007.

[38] J. F. Cavanagh, M. X. Cohen, and J. J. B. Allen, "Prelude to and resolution of an error: EEG phase synchrony reveals cognitive control dynamics during action monitoring," Journal of Neuroscience, vol. 29, no. 1, pp. 98-105, 2009. 